\title{
Psychological mechanisms in a digital intervention to improve physical activity: A multicentre randomized controlled trial
}

\author{
Ralf Schwarzer ${ }^{1,2}$ (D) , Lisa Warner', Lena Fleig ', Maryam Gholami ', \\ Sergio Salvatore ${ }^{3}$, Luisella Cianferotti ${ }^{4}$, Evangelia Ntzani ${ }^{5,6}$, \\ Blanca Roman-Viñas ${ }^{7,8}$, Antonia Trichopoulou ${ }^{9}$ and Maria L. Brandi ${ }^{4}$ \\ 'Freie Universität Berlin, Germany \\ ${ }^{2}$ SWPS University of Social Sciences and Humanities, Wroclaw, Poland \\ ${ }^{3}$ Euro-Mediterranean Biomedical Scientific Institute, University of Salento, Lecce, Italy \\ ${ }^{4}$ Department of Surgery and Translational Medicine, University of Florence, Italy \\ ${ }^{5}$ Department of Hygiene and Epidemiology, University of loannina School of Medicine, \\ Greece \\ ${ }^{6}$ Center for Evidence-Based Medicine, Department of Health Services, Policy and \\ Practice, School of Public Health, Brown University, Providence, Rhode Island, USA \\ ${ }^{7}$ Nutrition Research Foundation, University of Barcelona Science Park, Spain \\ ${ }^{8}$ CIBER Physiopathology of Overweight and Nutrition (CIBERobn), Institute of Health \\ Carlos III (ISCIII), Madrid, Spain \\ ${ }^{9}$ Hellenic Health Foundation, Athens, Greece
}

Objectives. The randomized controlled trial examined factors that might be responsible for individual differences in physical activity change among men and women who participated in a lifestyle intervention. The main purpose of the analyses regarded the role of psychological mechanisms involving motivation, planning, self-monitoring, and habit strength.

Design. A two-arm digital intervention was conducted in Italy, Spain, and Greece to improve physical activity levels, with follow-ups at 3 and 6 months after baseline assessment.

Methods. Participants were I,564 adults at baseline, $n=638$ at 6-month follow-up. Linear mixed models examined the intervention effects, and a two-group longitudinal structural equation model explored which psychological constructs (motivation, planning, self-monitoring, habit strength) were associated with changes in physical activity.

Results. In addition to an overall increase in self-reported activity, there were interactions between time and sex and between time and experimental groups, and a triple interaction between time, sex, and experimental groups, indicating that men reported an increase in activity independent of groups, whereas women in the active control group did not benefit from the intervention. Planning, self-monitoring, and habit strength mediated sequentially between initial motivation and follow-up physical activity.

*Correspondence should be addressed to Ralf Schwarzer, Freie Universität Berlin, Habelschwerdter Allee 45, Berlin D- I4I95, Germany (email: health@zedat.fu-berlin.de). 
Conclusions. Although the intervention produced overall improvements in physical activity, the time-by-treatment interaction emerged only for women. The mechanism included a sequence leading from motivation via planning, self-monitoring, and habit strength towards physical activity.

\section{Statement of contribution}

What is already known on this subject?

- Digital lifestyle interventions can be effective in terms of physical activity performance gains.

- Men are on average more physically active than women.

- Long-term adherence rates to digital interventions are usually low.

\section{What does this study add?}

- Giving users of an online platform more interactive options did not make a difference.

- Women gained more than men from adaptive, dynamic online platform content.

- Individual characteristics (motivation, planning, self-monitoring, habit) were more important than online treatment features.

According to the Eurobarometer Survey (European Commission, 2014), only $41 \%$ of citizens in Europe exercise or play sports at least once a week, whereas 59\% never or seldom do so. Some other form of physical activity (such as cycling, dancing, or gardening) is performed by $48 \%$ at least once a week, whereas $30 \%$ never do so. Overall, men are more active than women, which is mainly due to differences within the younger age group. Young men are considerably more active than young women ( $74 \%$ vs. $55 \%)$. Regular activity decreases with age, as $71 \%$ of women and $70 \%$ of men in the $55+$ age groups never or seldom exercise or play sports. The lowest levels of exercise participation are found in the Mediterranean countries such as Italy, Spain, or Greece (European Commission, 2014) where this study originates.

There are a large number of lifestyle change programmes that address this issue. In a review by Afshin et al. (2016), 29 randomized controlled trials on digital physical activity promotion were reviewed, resulting in clear evidence for their overall effectiveness. The authors conclude that Internet interventions improve lifestyle behaviours up to 1 year. On the other hand, Allman-Farinelli et al. (2016) evaluated maintenance outcomes of a 3-month digital intervention on prevention of weight gain and lifestyle behaviours at 9 months from baseline and found no differences in physical activity levels. Kwasnicka, Dombrowski, White, and Sniehotta (2016) argue that evidence for the sustainability of behaviour change in response to interventions is limited and this may be due to a lack of theoretical elaboration of the maintenance phase of behaviour change. These authors recommend a focus on self-regulatory constructs and habit formation.

This intervention study comprises psychological constructs and behaviour change strategies. Inspired by the health action process approach (Schwarzer, 2008), behavioural intentions, self-monitoring, and planning as key self-regulatory processes are assumed to operate jointly to translate motivation into habitual action.

\section{Motivation}

Forming a behavioural intention represents an indicator of motivation to change behaviours. For example, individuals may commit themselves to be more active in the near future and express their inclination to make lifestyle changes. By expressing an explicit behavioural intention, they are motivated to act, although such motivation does not necessarily need to be translated into actual behaviours if barriers emerge. This renders the intention instable. 


\section{Planning}

Motivation is more likely to be translated into behaviours when people generate detailed plans, imagine success scenarios, and develop preparatory strategies of tackling a challenging task. Planning is a prospective self-regulatory skill where an individual specifies the situational context in which one will enact to ensure that behavioural performance is achieved. Behavioural intentions are more likely to be translated into action when people develop preparatory strategies, such as making action plans of approaching a difficult task (Barz et al., 2014). Planning can easily be communicated to individuals with self-regulatory deficits, and it is frequently applied in health behaviour change interventions (for a review, see Hagger \& Luszczynska, 2014).

\section{Self-monitoring}

Self-monitoring is the key component of action control. While planning is a prospective strategy - that is, behavioural plans are made before the situation is encountered - selfmonitoring is a concurrent self-regulatory strategy, where the ongoing behaviour is continuously evaluated in terms of a behavioural standard (Sniehotta, Scholz, \& Schwarzer, 2006). Self-monitoring appears to be essential in the adoption and early maintenance of health behaviours (Greaves et al., 2011). Later, when the behaviour becomes habitual, conscious self-monitoring is no longer needed because behavioural engagement becomes automatic.

\section{Habit strength}

Self-monitoring and habit formation represent two sequential processes in the course of behaviour change, the latter one being proof of successful adoption (Gardner, 2015). Automaticity makes self-monitoring redundant. Habitual action originates in a decision to act, and subsequently, as a result of frequent repetition, acquires the characteristics of automaticity over time (e.g., Fleig, Pomp, Parschau, et al., 2013; Fleig, Pomp, Schwarzer, \& Lippke, 2013; van Bree et al., 2016).

\section{Aims}

This randomized controlled trial examined changes in physical activity levels among Southern European men and women who had attended one of two arms of a digital lifestyle intervention. It was hypothesized that on average, participants would increase their physical activity levels (time effect) and that men would be more active than women (sex effect). Moreover, it was hypothesized that participants in the experimental group would progress further than participants in the control group (time $\times$ treatment effect). The main exploratory purpose of the analyses, however, regarded the role of psychological mechanisms that might have been responsible for individual differences in the process of behaviour change. The psychological constructs involved were motivation, planning, self-monitoring, and habit strength. It was assumed that initial motivation at the onset of the intervention made a difference for all subsequent processes, thus influencing the likelihood of planning as well as the eventual success of the intervention, as reflected by higher physical activity levels. It was also assumed that planning and self-monitoring would affect subsequent habit strength, which, in turn, would be associated with physical activity. Although this chain of operating constructs appeared to be theoretically meaningful, it remained to be explored whether the 
hypothesized sequence showed up in this particular sample, and which pattern of direct and indirect effects would emerge. Thus, in addition to the expected intervention effect, the possible psychological mechanism was being examined, confirming a theoretical sequence and exploring in more detail the pattern of effects.

\section{Method}

\section{Participants and procedure}

This two-arm randomized controlled trial targeted adult residents in Italy, Greece, and Spain from 2015 to 2016 as part of the Credits4Health Project ('Credits-based, peoplecentric approach for the adoption of healthy life-styles and balanced Mediterranean diet in the frame of social participation and innovation for health promotion', funded by the European Commission within the VII Framework Program, Grant Agreement: 602386). The project developed an online platform with personalized motivational pathways to improve diet and activity levels in the three Mediterranean countries. A full description of the trial is provided in the Data S1.

Potential participants were recruited through radio messages and Web announcements, as well as through use of local mailing lists. We obtained ethical approval from all local review boards. Individuals were eligible to participate if they were apparently healthy, at least 18 years old, and residing in one of the selected areas in Italy, Spain, and Greece. A total of 2,064 persons were screened and provided with information about the purpose of the study. After giving informed consent, they received an account for the online platform to complete a baseline questionnaire with behavioural and psychological items that was attended by 1,569 individuals ( 951 women, 618 men). Participants were randomized to either the experimental (dynamic platform) or the active control group (static platform) by a computer algorithm, and they were informed about their group membership, indicating that those in the active control group would have the option to benefit from the experimental treatment after completion of the study. There was no passive control group. After 3 and 6 months, they received a notice on their personal online dashboard, asking them to fill out the follow-up assessments. In the longitudinal sample to be analysed, after attrition, 323 persons remained in the control group, and 315 persons remained in the experimental group (Figure 1).

\section{Intervention}

The online platform delivered a lifestyle intervention to improve physical activity and dietary habits over a 6-month period that implemented theory-based behaviour change components. In this study, we focus on the physical activity components of the complex intervention. The two groups differed in terms of the amount and type of options that were available to them at the online platform (see Supporting Information).

\section{Dynamic platform}

The experimental group had access to a dynamic platform that was built to deliver personalized paths according to self-set goals and individual characteristics along with rewards for achieved behavioural outcomes. The dynamic platform was characterized by several interactive features, including personalized feedback based on their nutritional habits, updates and prompts about their physical activity status, reward messages based on the goals they set, and credits awarded based on their set goals and achievement thereof. 


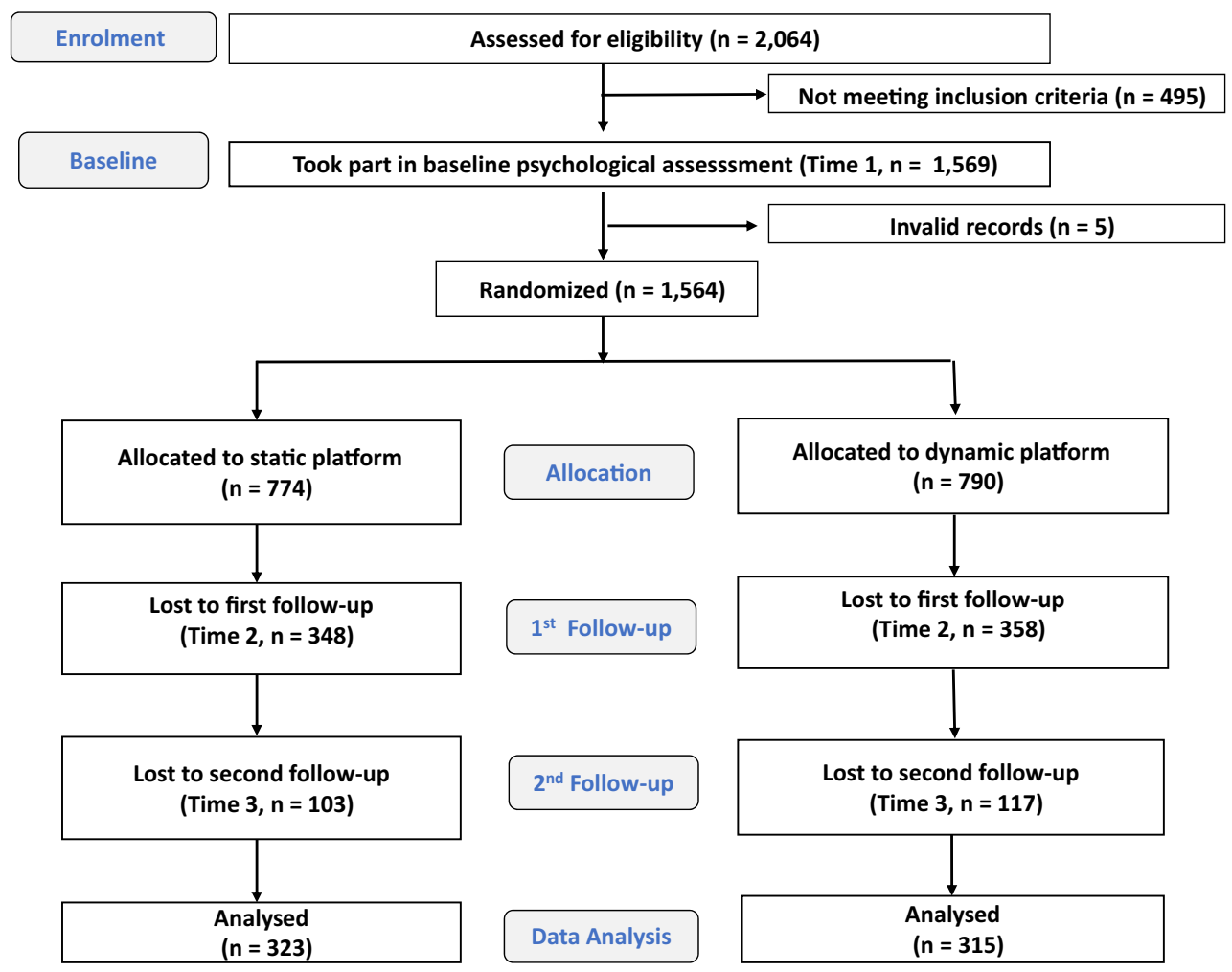

Figure I. Flow diagram outlining participant allocation into the experimental group (dynamic online platform) or the active control group (static online platform).

The active treatment delivered through the dynamic platform began right after the randomization, considering the information entered by the recruiters and self-reported by participants. An algorithm provided personalized advice on nutrition and physical activity to help individuals meet their self-imposed goals. Participants in the experimental group set their goals by choosing between weekly amounts of calories to be burnt (tailored to their specific physical status), and between different paths to attain their weekly goal (easy, medium, or challenging path). Based on the goals and paths set, the user could choose the activity, the duration, and the intensity to meet the planned caloric expenditure, and plan a weekly schedule accordingly. Users then reported their performed activities, receiving positive feedback if they were in line with the plan, or being asked to analyse the reasons for setbacks. The platform system provided suggestions and strategies to overcome barriers to behaviour change and encouraged them to reschedule the activities. The general aims were to help users in setting their goals, in assessing whether they manage to plan and perform them, and in supporting them towards higher goals in case of success or helping them understand and tackle the reasons for setbacks.

\section{Static platform}

The active control group had access to a static platform that was characterized by provision of detailed, non-personalized information on proposed nutritional and 
physical activity habits. No personalized feedback, goal-setting, or credits were provided. The static platform served as a data repository and provided information on recommended health behaviours according to participants' baseline profile. The active control participants were informed that they could switch to the dynamic platform after completion of the study and end of the pre-defined 6month follow-up, which means that they were not blinded to the existence of two conditions.

\section{Measures}

All measures were administered in Italian, Spanish, Catalan, and Greek languages. The psychological constructs motivation, planning, and self-monitoring were all assessed with items adapted from Sniehotta, Scholz, and Schwarzer (2005) rated on a 6-point Likert scale ranging from (1) not at all true to (6) exactly true.

\section{Physical activity indices}

As an indicator for levels of physical activity at all three points in time, the General Practice Physical Activity Questionnaire (GPPAQ) was used, which is a common screening tool that generates a sum score index and a categorical index (Department of Health, 2009). The questionnaire asks about heterogeneous types and amounts of physical activity at work and leisure time, including amount of walking, cycling, housework, and gardening. This instrument had also been validated in Spanish and Catalan (Puig Ribera et al., 2012). The sum score for the GPPAQ ranges from 0 (lowest) to 8 (highest), and it had a 3-month retest reliability of $r_{\mathrm{tt}}=.69$. It also provides a four-level physical activity index of being active (4), moderately active (3), moderately inactive (2), and inactive (1). Retest reliability of the index over 3 months: $r_{\mathrm{tt}}=.62, p<.01$. Intercorrelation of both variables is $r=.87$, $p<.01$.

\section{Motivation index}

Activity-specific motivation at baseline was assessed by an index based upon three items that pertained to vigorous, moderate, and light exercise, namely 'I have a strong commitment to (a) ... vigorously exercise regularly, so that I sweat and become short of breath, (b) . . . be regularly and moderately active, so that I sweat a bit in leisure time, (c) ... be active in daily life (walking, biking, house and garden work)'. Retest reliability of the index over 3 months: $r_{\mathrm{tt}}=.52, p<.01$.

\section{Planning scale}

Activity-specific planning was assessed at Time 2 by four items, two of them pertaining to action planning with the stem 'I have made concrete and detailed plans ...' followed by the items (a) '... how, when and where I will be physically active' and (b) '... how often and with whom to exercise'. The other two items referred to coping planning with the response options (c) '...which alternative activity I will choose, in case I cannot perform my originally planned activity' and (d) '...what to do instead, if I do not have the time or if any other obstacle or interruption emerges'. Cronbach's $\alpha$ was .91. 


\section{Self-monitoring scale}

Activity-specific self-monitoring was assessed at Time 2 with the following three statements: (a) 'I have monitored how active I was in terms of how often, how long, and which intensity, (b) I have monitored how often and how long I have NOT been active, (c) I have kept records about the amount of my activity'. Cronbach's $\alpha$ was .72.

\section{Habit strength scale}

Activity-specific habit strength was assessed by four items of the Self-Report Behavioural Automaticity Index (Gardner, Abraham, Lally, \& de Bruijn, 2012) that pertain to the automaticity of being physically active, namely 'Being physically active...' (a) '...is something I do without thinking', (b) '.. is something I do automatically', (c) '....is something I start doing before I realize I'm doing it', and (d) ' $\ldots$ is something I do without having to consciously remember'. Cronbach's $\alpha$ was .97.

\section{Data analysis}

Two pre-planned approaches were chosen in line with the research questions. First, mean level changes of physical activity over three points in time were examined dependent on experimental conditions, sex, and motivation. Linear multilevel models were computed using the SPSS 24 MIXED procedure (Heck, Thomas, \& Tabata, 2014; Hoffman, 2015). We specified the time-varying GPPAQ sum score as level 1 dependent variable with three time points crossed in individuals (level 2) with restricted maximum-likelihood estimation which also accounts for missing values in the dependent variable. Motivation, age, and sex served as level 2 time-invariant covariates. We studied cross-level interactions to determine the interrelationships between age (grand-mean-centred), motivation (grandmean-centred), sex (coded as 1 [men], 0 [women]), and time points (baseline $=0$, intermediate $=1$, final assessments $=2$ ). In a linear mixed-effects model, the responses from participants are thought to be the sum of fixed and random effects. The fixed effects (model for the means) are of primary interest, and random effects contribute to the covariance structure of the data. Adjustments for the covariance structure make the results more accurate. An unstructured covariance matrix for random intercepts and random time effects was chosen. As an effect size estimate, the total $R^{2}$ was computed, which is the squared correlation between the actual outcome and the outcome predicted by the fixed effects. A series of analyses aimed at identifying a suitable model for the variances by comparing fit indices, mainly the Akaike's Information Criterion (AIC). The analysis had sufficient statistical power. With an alpha level of .05 and a stability factor of .50 , a minimum sample size of only $N=306$ would be required to detect a small effect, $f(V)=0.2$, partial $\eta^{2}=.04$, with a power of .90 .

The second approach to data analysis was the examination of possible psychological mechanisms that may be responsible for individual differences in behaviour change. For this purpose, a longitudinal structural equation model (SEM) was chosen with follow-up physical activity as the final outcome to be predicted by the baseline information (sex, age, motivation, experimental conditions) and further qualified by subsequent psychological constructs (planning, self-monitoring, habit strength) specified as sequential mediators. Computations were carried out with Mplus 7.4 (Muthén \& Muthén, 1998-2015). As a database, the sample of those who had values on all model variables was chosen $(N=590)$. Model fit was evaluated in terms of the comparative fit index (CFI) and the root mean square error of approximation (RMSEA). 


\section{Results}

\section{Preliminary descriptive and attrition analyses}

Baseline physical activity data were available from 1,569 individuals, 858 of them attended the intermediate test after 3 months, and 638 completed the follow-up assessment after 6 months. These constituted the final longitudinal sample (see Figure 1). In this subsample, initial average age was 43.01 years $(S D=10.82$; range: $19-66$ years), and initial average body mass index was $25.59(S D=4.65$; range: $16.7-49.01)$. Randomization was based on 1,564 individuals, of whom 774 were invited to log on to the static online platform, whereas 790 were invited to log on to the dynamic platform. At the last followup assessment (Time 3), $n=323$ individuals (137 men, 186 women) were available for data analyses in the static platform, compared to $n=315$ persons ( 120 men, 195 women) in the dynamic platform.

The means, standard deviations, and intercorrelations between the main study variables are presented in Table 1 . The average GPPAQ sum score increased over time, with sex differences as men scored higher than women, although women reported higher habit strength at Time 3. There were no sex differences in terms of age, T1 motivation, T2 planning, and T2 self-monitoring. Physical activity was significantly correlated with all other variables, and closest with T2 planning. The association of physical activity with age was negative. This means that individuals who were somewhat younger than average were more active.

Analyses of variance were computed with study dropout as the independent variable and the baseline assessments as dependent variables (age, sex, motivation). The result indicated that those who remained in the study at final follow-up differed on age and motivation compared to those who dropped out: On the 1-6 range, remainers scored slightly higher $(M=4.43, S D=0.97)$ on initial motivation than dropouts $(M=4.29$, $S D=0.95, p<.01$ ) and were on average 4 years older (remainers: $M=43.02$ years, $S D=11.8$, dropouts: $M=39.25$ years, $S D=10.8, p<.01)$.

\section{Mean level changes in physical activity and differential effects on changes}

The analyses aimed at testing the effects on physical activity as measured by the GPPAQ sum score. Linear mixed models were computed with time points nested in individuals, using the GPPAQ sum score at three time points as the level 1 dependent variable. Individual differences in terms of age, motivation, and sex served as time-invariant covariates at level 2 . First, the intraclass correlation was computed, finding that $71 \%$ of the entire physical activity variance was due to interindividual differences at level 2 , whereas $29 \%$ was at level 1, within person, for example, time-specific deviations around one's mean level. The findings (mixed model for the means) are presented in Table 2 and Figure 2 . The variance accounted for by the final model was a total $R^{2}=.15$. Experimental groups did not differ at baseline $(b=-0.02, p=.75)$. Time showed an increasing overall trend $(b=.18, p<.01)$. Sex $(b=0.71, p=<.01)$ was associated with the initial levels of GPPAQ. Age $(b=-0.03, p=<.01)$, and initial motivation $(b=0.49, p=<.01)$ reflected individual differences, which means that somewhat younger-than-average adults and those with higher motivation were more likely to report higher physical activity levels. There was no significant covariance between intercept and slope (Wald $z=-1.23$, $p=.22)$. These findings were qualified by cross-level interactions between time and experimental groups $(b=0.23, p=<.01)$ and between time and $\operatorname{sex}(b=0.18, p=.03)$, and a triple interaction between time, sex, and experimental groups $(b=-0.31$, 


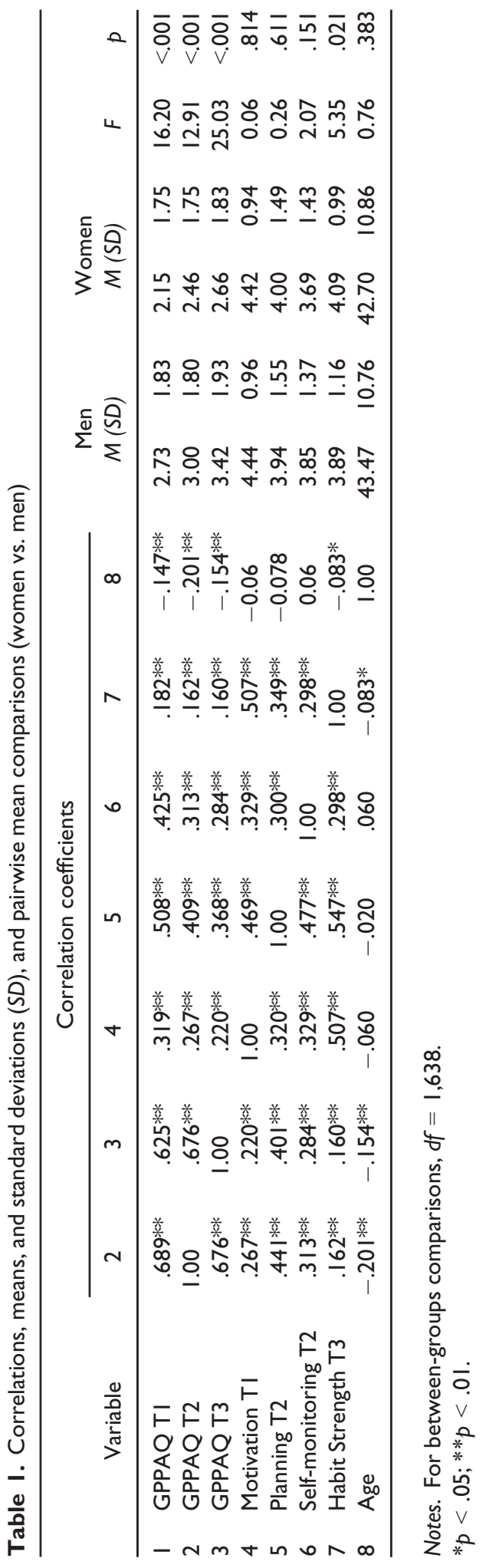


Table 2. Results of linear mixed modelling: Dependent variable is physical activity (GPPAQ sum score) at three points in time. Model for the means in the upper panel and model for the variance in the lower panel

\begin{tabular}{|c|c|c|c|c|c|c|c|c|}
\hline \multirow{2}{*}{\multicolumn{2}{|c|}{ Parameter }} & \multirow[b]{2}{*}{ Estimate } & \multirow[b]{2}{*}{ SE } & \multirow[b]{2}{*}{$d f$} & \multirow[b]{2}{*}{$t$} & \multirow[b]{2}{*}{$p$} & \multicolumn{2}{|c|}{ 95\% Confidence interval } \\
\hline & & & & & & & Lower bound & Upper bound \\
\hline \multicolumn{2}{|l|}{ Intercept } & 3.24 & 0.16 & 1608.05 & 20.73 & $<.01$ & 2.94 & 3.55 \\
\hline \multicolumn{2}{|c|}{ Time } & 0.18 & 0.05 & 840.96 & $3.4 I$ & $<.01$ & 0.07 & 0.28 \\
\hline \multirow{2}{*}{\multicolumn{2}{|c|}{$\begin{array}{l}\text { Experimental Groups } \\
\operatorname{Sex}(\text { male }=1, \text { female }=0)\end{array}$}} & -0.03 & 0.08 & 1554.15 & -0.32 & .75 & -0.19 & 0.14 \\
\hline & & 0.71 & 0.09 & 1554.15 & 8.16 & $<.01$ & 0.54 & 0.88 \\
\hline \multicolumn{2}{|c|}{ Age } & -0.03 & 0.00 & 1564.93 & -8.22 & $<.01$ & -0.04 & -0.02 \\
\hline \multicolumn{2}{|l|}{ Motivation } & 0.49 & 0.04 & 1542.62 & 11.66 & $<.01$ & 0.41 & 0.57 \\
\hline \multicolumn{2}{|l|}{ Time $\times$ Groups } & 0.23 & 0.07 & 83242 & 3.25 & $<.01$ & 0.09 & 0.37 \\
\hline \multirow{2}{*}{\multicolumn{2}{|c|}{$\begin{array}{l}\text { Time } \times \text { Sex } \\
\text { Time } \times \text { Sex } \times \text { Groups }\end{array}$}} & 0.18 & 0.08 & 81577 & 2.24 & .03 & 0.02 & 0.33 \\
\hline & & -0.31 & 0.11 & 767.66 & -2.83 & $<.01$ & -0.52 & -0.09 \\
\hline \multirow{2}{*}{\multicolumn{3}{|c|}{ Parameter }} & \multirow[b]{2}{*}{ Estimate } & \multirow[b]{2}{*}{$S E$} & \multirow[b]{2}{*}{ Wald Z } & \multirow[b]{2}{*}{$p$} & \multicolumn{2}{|c|}{$95 \%$ Confidence interval } \\
\hline & & & & & & & Lower bound & Upper bound \\
\hline \multirow[t]{3}{*}{ Repeated Measures } & \multicolumn{2}{|c|}{ Var: $($ Time $=0)$} & 0.79 & 0.15 & 5.23 & $<.01$ & 0.54 & 1.14 \\
\hline & \multicolumn{2}{|c|}{ Var: $($ Time $=1)$} & 1.04 & 0.09 & 12.13 & $<.01$ & 0.88 & 1.22 \\
\hline & \multicolumn{2}{|c|}{ Var: $($ Time $=2)$} & 1.00 & 0.17 & 5.75 & $<.01$ & 0.71 & 1.40 \\
\hline \multirow[t]{3}{*}{ Intercept + Time } & \multicolumn{2}{|c|}{ UN $(I, I)$} & 2.06 & 0.16 & 12.50 & $<.01$ & 1.76 & 2.41 \\
\hline & UN $(2$ & & -0.12 & 0.10 & -1.23 & .22 & -0.32 & 0.07 \\
\hline & UN $(2$ & & 0.17 & 0.08 & 2.14 & .03 & 0.07 & 0.43 \\
\hline
\end{tabular}

$p=<.01$ ). While men were overall more active than women, study participants increased their physical activity over time, which was in particular true for women in the experimental group (dynamic online platform) as compared to women in the active control group (static online platform).

\section{Psychological mechanisms in the process of physical activity change}

The previous analyses have uncovered the interplay of experimental conditions with baseline motivation and sex. At follow-up, other psychological variables were available that allowed for a closer look at the possible mechanisms that may be responsible for changes in physical activity levels. Such mechanisms were examined in the context of longitudinal SEM. Sex has been established as a moderator, comparing men and women by a two-group model.

To predict physical activity levels at the final follow-up (Time 3), baseline activity was specified as a covariate along with initial motivation, whereas planning and selfmonitoring at Time 2 and habit strength at Time 3 were specified as sequential mediators. The rationale behind this was the assumption of a sequence, starting with study entry characteristics and experimental treatment, followed by planning and self-monitoring which reflect intervention content, and by habit strength as a result of the previous steps.

A two-group longitudinal SEM with multiple-indicator latent variables was specified with physical activity as a latent variable at Time 3 follow-up controlling for its baseline counterpart. This construct was based on two indicators, namely the GPPAQ sum score 


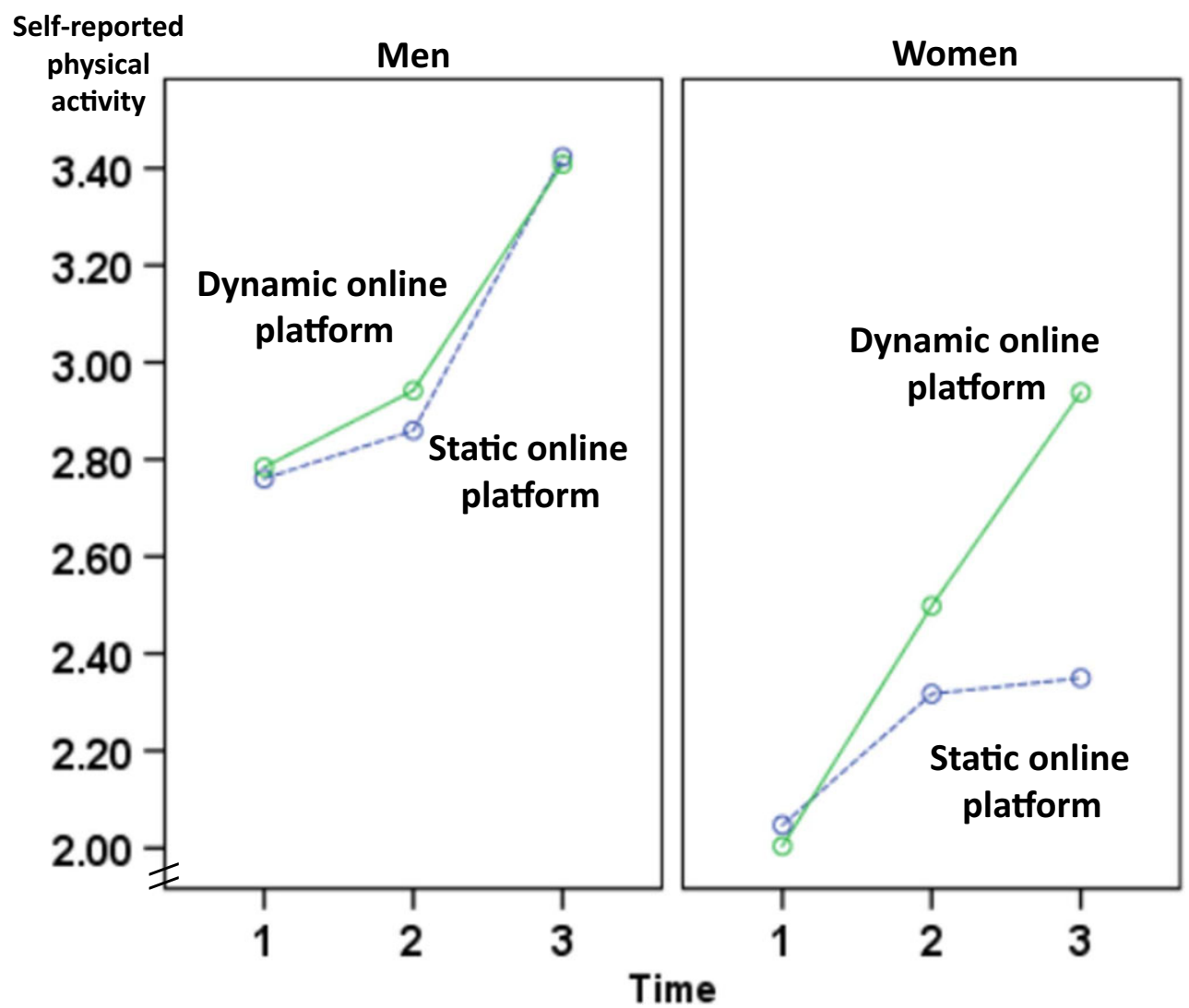

Figure 2. Mean level changes in self-reported physical activity under two experimental conditions, moderated by sex. Dependent variable is physical activity (GPPAQ sum score) at three points in time with a range from 0 to 8 ( $N=638$ adults with complete longitudinal GPPAQ data).

and the GPPAQ index $(r=.87, p<.01)$. T1 motivation, T2 planning, T2 self-monitoring, and $\mathrm{T} 3$ habit strength as latent variables included their corresponding items as multiple indicators. All factor loadings exceeded the level of 0.40. Age was supposed to affect baseline activity, as confirmed by the previous mixed model analyses. Intervention conditions were supposed to affect T2 planning, because planning tasks were given as intervention components, which means that study participants on the dynamic online platform (treatment group) should develop better planning skills. Intervention conditions, initial motivation, and baseline activity were specified as exogenous variables, follow-up activity as endogenous variable to be predicted, and planning, self-monitoring, and habit strength also as endogenous variables representing sequential mediators. Listwise deletion of missing values resulted in a sample size of $n=590$ individuals who had complete data across all three measurement points in time. Total sample analyses $(N=1,564)$ using the full-information maximum-likelihood procedure to account for missing values yielded similar parameter estimates but are not reported because iterations did not converge. The model fit to the data was satisfactory with $\chi^{2}(338)=785.39$, $p<.01, \mathrm{CFI}=.95$, and RMSEA $=.06$.

Physical activity at follow-up was predicted jointly by habit strength, planning, and baseline activity (Figure 3). The psychological mediators operated in a sequential manner, 


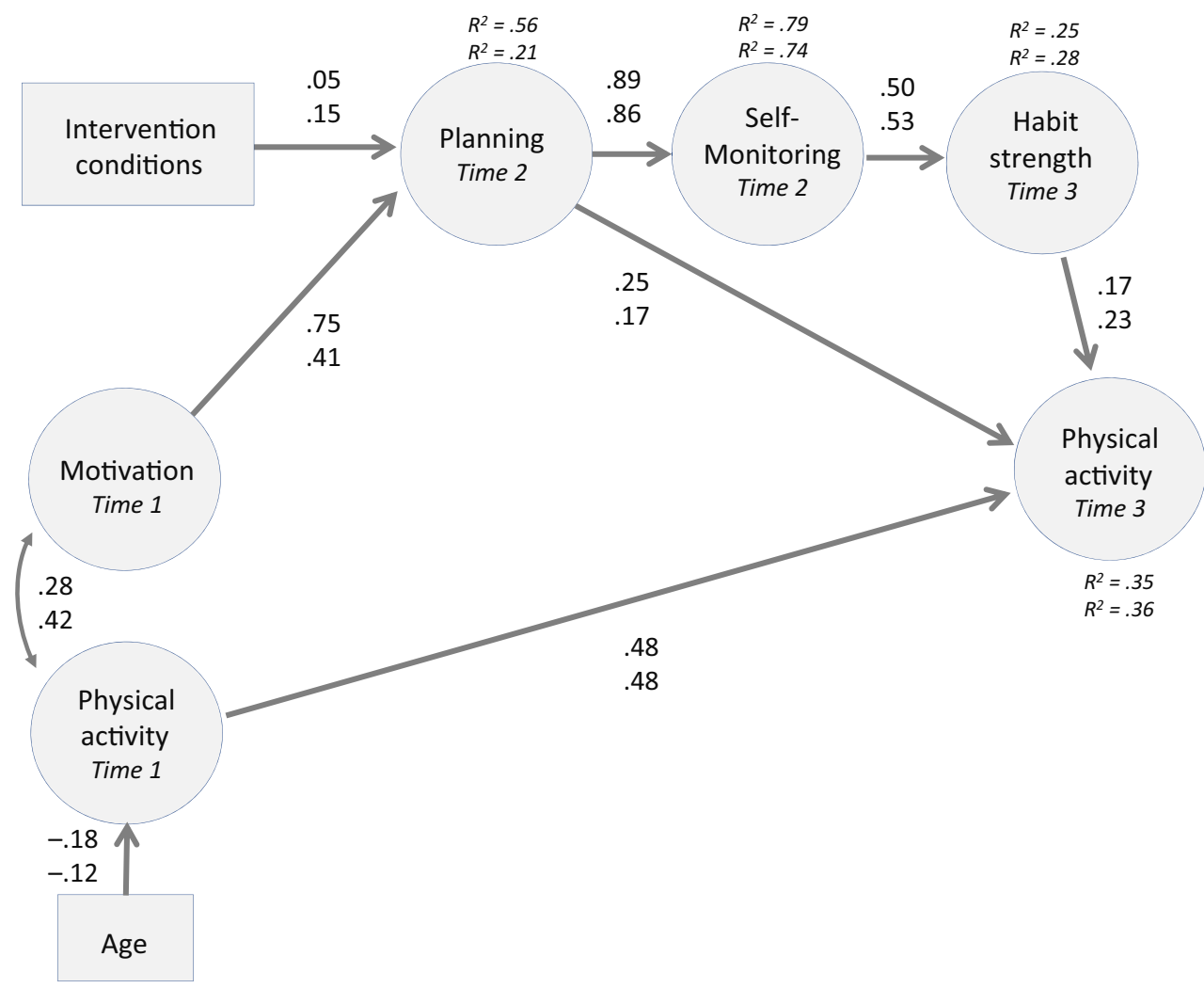

Figure 3. Two-group multiple-indicator structural equation model examining psychological mechanisms of physical activity change ( $N=590$ adults with data on included variables).

Note: Standardized parameter estimates, upper coefficients: men; lower coefficients: women.

starting from initial motivation via planning and self-monitoring to habit strength. It is of note that intervention conditions did not yield an overall or direct effect on any latent construct except of planning. This means that treatment groups had only a small indirect effect on final physical activity. Most relevant are the sex differences that emerged when predicting planning at $\mathrm{T} 2$. Only for women, planning was predicted by the intervention, whereas men's plans were unaffected by study groups. In men, the planning levels were, however, determined by their initial motivation.

\section{Discussion}

An overall increase in physical activity was observed, although a causal attribution of this change to the treatment cannot be proven, due to the lack of a passive control group. The increase was qualified by associations with sex such as that men started at a higher activity level than women, and their increase over time was independent of their membership in the two intervention arms. The desired time-by-treatment interaction was due to the women only, who benefitted from the dynamic online platform (treatment group), whereas they hardly changed their activity levels when being randomized to the static platform (active control group). 
The main aim of these analyses was to examine the possible psychological mechanisms that may be responsible for individual differences in activity changes. The two-group longitudinal SEM confirmed the theoretical assumptions. In line with this, initial motivation made planning more likely, which was closely associated with more selfmonitoring of physical activity, and this, in turn, led to more habitual activity. Initial motivation was not directly associated with physical activity later on, which is in line with other studies such as the one by Maher and Conroy (2016), who did not find a link between behavioural intention on the one hand and habit strength and light-intensity exercise on the other. They argue that cognitively controlled processes operate rather independently from automatic processes, as reflected by habitual behaviours (see also Borland, 2017). Although the overall sequential mediation was the same for men and women, there were also notable differences at the onset of the chain of cognitive processes leading to activity. As planning and self-monitoring were essential components of the digital intervention, it was expected that the treatment would be most influential on these two processes at follow-up. However, for men such an intervention effect was not significant at all. Their levels of planning were determined by their initial motivation to exercise. For women, being assigned to the dynamic or static online platform, however, made a difference to their plans. For them, formulating weekly activity plans in the online calendar seems to have increased their planning processes over time. Stronger effects of self-regulatory intervention on the planning efforts of women have also previously been reported in physical activity trials (Hankonen, Absetz, Ghisletta, Renner, \& Uutela, 2010). These authors interpret their findings by stating that women might need more planning to integrate physical activity into their daily lives, as they receive less social support, experience less acceptance of lifestyle changes in their social surrounding, and have a lower chance of making spontaneous lifestyle decisions. This is also in line with previous observational studies showing that women, generally, use fewer planning strategies when it comes to engaging in physical activity (e.g., Arnautovska, Fleig, O'Callaghan, \& Hamilton, 2017).

In previous research, planning and self-monitoring have been identified as important proximal predictors of behaviours (Hagger \& Luszczynska, 2014), whereas in the present study, this is qualified by habit strength as the most proximal predictor. This is a meaningful result as it underscores the process nature of physical activity change. Planning and self-monitoring are mainly necessary, when adopting a higher level of exercise but less so later on, when the behaviour becomes more habitual. Accordingly, planning and self-monitoring should become less predictive of physical activity as habit strength increases, so that where planning and self-monitoring are weak and habit is strong, behaviour corresponds with habit and not the other self-regulatory constructs.

The findings of the current study need to be interpreted in the light of their possible limitations. Demand characteristics due to self-reporting and recall bias due to retrospective assessment of behaviour may have biased the reporting of activities. The digital two-arm intervention has resulted in an overall increase in physical activity in those participants who remained in the study at 6-month follow-up. One can speculate that those who have dropped out prematurely may have been less successful. The attrition rate is not surprising but commonly experienced by researchers in the area of digital interventions, a phenomenon also called the 'law of attrition' (Eysenbach, 2005). Due to the lack of control over active participation and the easiness of withdrawal, dropout rates are usually high in online interventions.

In conclusion, the current study demonstrates differential intervention effects, pointing also to the role of gender in dietary change, and it suggests a theory-inspiring 
sequential mediation chain that sheds light on the psychological mechanisms of lifestyle changes. Given the sex-specific processes highlighted in our study, future intervention programmes should consider tailoring their intervention materials to men and women. Tailoring can be done by frequent use of testimonials in which men or women describe how they have made activity plans and how they have coped with temptations and setbacks. Self-disclosing coping models tend to generate self-efficacy in observers. Future research should target the tailoring of digital interventions to subgroups, employ more refined assessments of physical activity, and extend the follow-up period to more than half a year.

\section{Conflict of interest}

All authors declare no conflict of interests.

\section{References}

Afshin, A., Babalola, D., McLean, M., Yu, Z., Ma, W., Chen, C. Y., ... Mozaffarian, D. (2016). Information technology and lifestyle: A systematic evaluation of internet and mobile interventions for improving diet, physical activity, obesity, tobacco, and alcohol use. Journal of the American Heart Association, 5(9), e003058. https://doi.org/10.1161/jaha.115.003058

Allman-Farinelli, M., Partridge, S. R., McGeechan, K., Balestracci, K., Hebden, L., Wong, A., ... Bauman, A. (2016). A mobile health lifestyle program for prevention of weight gain in young adults (TXT2BFiT): Nine-month outcomes of a randomized controlled trial. JMIR mHealth and uHealth, 4(2), e78. https://doi.org/10.2196/mhealth.5768

Arnautovska, U., Fleig, L., O'Callaghan, F., \& Hamilton, K. (2017). A longitudinal investigation of older adults' physical activity: Testing an integrated dual-process model. Psychology \& Health, 32, 166-185. https://doi.org/10.1080/08870446.2016.1250273

Barz, M., Parschau, L., Warner, L. M., Lange, D., Fleig, L., Knoll, N., \& Schwarzer, R. (2014). Planning and preparatory actions facilitate physical activity maintenance. Psychology of Sport and Exercise, 15, 516-520. https://doi.org/10.1016/j.psychsport.2014.05.002

Borland, R. (2017). CEOS Theory: A comprehensive approach to understanding hard to maintain behaviour change. Applied Psychology: Health and Well-Being, 9(1), 3-35. https://doi.org/10. 1111/aphw.12083

Department of Health. (2009). The General Practice Physical Activity Questionnaire: A screening tool to assess adult physical activity levels, within primary care. Retrieved from www.gov. uk/government/uploads/system/uploads/attachment_data/file/192453/GPPAQ_-_guidance. pdf

European Commission. (2014). Special Eurobarometer 412 - Sport and physical activity report. Retrieved from www.ec.europa.eu/health/nutrition_physical_activity/docs/ebs_412_en.pdf

Eysenbach, G. (2005). The law of attrition. Journal of Medical Internet Research, 7(1), e11. https://doi.org/10.2196/jmir.7.1.e11

Fleig, L., Pomp, S., Parschau, L., Barz, M., Lange, D., Schwarzer, R., \& Lippke, S. (2013). From intentions via planning and behavior to physical exercise habits. Psychology of Sport Exercise, 14, 632-639. https://doi.org/10.1016/j.psychsport.2013.03.006

Fleig, L., Pomp, S., Schwarzer, R., \& Lippke, S. (2013). Promoting exercise maintenance: How interventions with booster sessions improve long-term rehabilitation outcomes. Rebabilitation Psychology, 58, 323-333. https://doi.org/10.1037/a0033885

Gardner, B. (2015). A review and analysis of the use of 'habit' in understanding, predicting and influencing health-related behaviour. Health Psychology Review, 9, 277-295. https://doi.org/ $10.1080 / 17437199.2013 .876238$ 
Gardner, B., Abraham, C., Lally, P., \& de Bruijn, G. J. (2012). Towards parsimony in habit measurement: Testing the convergent and predictive validity of an automaticity subscale of the Self-Report Habit Index. The International Journal of Behavioral Nutrition and Physical Activity, 9, 102. https://doi.org/10.1186/1479-5868-9-102

Greaves, C. J., Sheppard, K. E., Abraham, C., Hardeman, W., Roden, M., Evans, P. H., \& Schwarz, P. (2011). Systematic review of reviews of intervention components associated with increased effectiveness in dietary and physical activity interventions. BMC Public Health, 11, 119. https://doi.org/10.1186/1471-2458-11-119

Hagger, M. S., \& Luszczynska, A. (2014). Implementation intention and action planning interventions in health contexts: State of the research and proposals for the way forward. Applied Psychology: Health and Well-Being, 6(1), 1-47. https://doi.org/10.1111/aphw.12017

Hankonen, N., Absetz, P., Ghisletta, P., Renner, B., \& Uutela, A. (2010). Gender differences in social cognitive determinants of exercise adoption. Psychology \& Health, 25(1), 55-69. https://doi. org/10.1080/08870440902736972

Heck, R. H., Thomas, S. L., \& Tabata, L. N. (2014). Multilevel and longitudinal modeling with IBM SPSS. New York, NY: Routledge.

Hoffman, L. (2015). Longitudinal analysis: Modeling within-person fluctuation and change. Oxford, UK: Routledge.

Kwasnicka, D., Dombrowski, S. U., White, M., \& Sniehotta, F. (2016). Theoretical explanations for maintenance of behaviour change: A systematic review of behaviour theories. Health Psychology Review, 10, 277-296. https://doi.org/10.1080/17437199.2016.1151372

Maher, J. P., \& Conroy, D. E. (2016). A dual-process model of older adults' sedentary behavior. Health Psychology, 35, 262-272. https://doi.org/10.1037/hea0000300

Muthén, L. K., \& Muthén, B. O. (1998-2015). Mplus user's guide (7th ed.). Los Angeles, CA: Author. Puig Ribera, A., Peña Chimenis, O., Romaguera Bosch, M., Duran Bellido, E., Heras Tebar, A., Solà Gonfaus, M., ... Cid Cantarero, A. (2012). How to identify physical inactivity in primary care: Validation of the Catalan and Spanish versions of 2 short questionnaires. Atencion Primaria, 44, 485-493. https://doi.org/10.1016/j.aprim.2012.01.005

Schwarzer, R. (2008). Modeling health behavior change: How to predict and modify the adoption and maintenance of health behaviors. Applied Psychology, 57(1), 1-29. https://doi.org/10. 1111/j.1464-0597.2007.00325.x

Sniehotta, F. F., Scholz, U., \& Schwarzer, R. (2005). Bridging the intention-behaviour gap: Planning, self-efficacy, and action control in the adoption and maintenance of physical exercise. Psychology \& Health, 20, 143-160. https://doi.org/10.1080/08870440512331317670

Sniehotta, F. F., Scholz, U., \& Schwarzer, R. (2006). Action plans and coping plans for physical exercise: A longitudinal intervention study in cardiac rehabilitation. British Journal of Health Psychology, 11(1), 23-37. https://doi.org/10.1348/135910705x43804

van Bree, R. J. H., Mudde, A. N., Bolman, C., van Stralen, M. M., Peels, D. A., de Vries, H., \& Lechner, L. (2016). Are action planning and physical activity mediators of the intention-habit relationship? Psychology of Sport and Exercise, 27, 243-251. https://doi.org/10.1016/j.psychsport.2016.09. 004

Received 2 I April 2017; revised version received I2 November 2017

\section{Supporting Information}

The following supporting information may be found in the online edition of the article:

Data S1. Publishable summary. 